\title{
Charge flipping vortices in the discrete nonlinear Schrodinger trimer and hexamer
}

\author{
Peter Jason and Magnus Johansson
}

\section{Linköping University Post Print}

\section{Tweet}

N.B.: When citing this work, cite the original article.

Original Publication:

Peter Jason and Magnus Johansson, Charge flipping vortices in the discrete nonlinear Schrodinger trimer and hexamer, 2015, Physical Review E. Statistical, Nonlinear, and Soft Matter Physics, (91), 2, 022910.

http://dx.doi.org/10.1103/PhysRevE.91.022910

Copyright: American Physical Society

http://www.aps.org/

Postprint available at: Linköping University Electronic Press

http://urn.kb.se/resolve?urn=urn:nbn:se:liu:diva-117256 


\title{
Charge flipping vortices in the discrete nonlinear Schrödinger trimer and hexamer
}

\author{
Peter Jason, ${ }^{*}$ and Magnus Johansson, ${ }^{\dagger}$ \\ Department of Physics, Chemistry, and Biology (IFM), Linköping University, SE-581 83 Linköping, Sweden
}

(Received 5 November 2014; published 13 February 2015)

\begin{abstract}
We examine the existence and properties of charge flipping vortices (CFVs), vortices which periodically flip the topological charge, in three-site (trimer) and six-site (hexamer) discrete nonlinear Schrödinger lattices. We demonstrate numerically that CFVs exist as exact quasiperiodic solutions in continuous families which connect two different stationary solutions without topological charge, and that it is possible to interpret the dynamics of certain CFVs as the result of perturbations of these stationary solutions. The CFVs are calculated with high numerical accuracy and we may therefore accurately determine many of their properties, such as their energy and linear stability, and the CFVs are found to be stable over large parameter regimes. We also show that, like in earlier studies for lattices with a multiple of four sites, trimer and hexamer CFVs can be obtained by perturbing stationary constant amplitude vortices with certain linear eigenmodes. However, in contrast to the former case where the perturbation could be infinitesimal, the magnitude of the perturbations for trimers and hexamers must overcome a quite large threshold value. These CFVs may be interpreted as exact quasiperiodic CFVs, with a small perturbation applied. The concept of a charge flipping energy barrier is introduced and discussed.
\end{abstract}

DOI: 10.1103/PhysRevE.91.022910

PACS number(s): 05.45.-a, 42.65.Sf, 42.65.Pc, 03.75.Lm

\section{INTRODUCTION}

Vortices are ubiquitous structures in systems described by complex fields [1], appearing in a wide variety of physical settings, such as superfluidity [2], superconductivity [3], BoseEinstein condensates (BECs) [4-6], and optics [7,8]. They are characterized by a topological phase dislocation, where the amplitude vanishes and around which the phase changes by a multiple of $2 \pi$. This multiple is called the topological charge (TC) or vorticity of the vortex, and is related to the flow of energy around the vortex core, with the sign indicating the direction. Since the TC only takes integer values, vortices may be of possible interest for applications, such as information storage [9] and communication [10].

The experimental progress in the last couple of decades within the fields of photonic lattices [11] and BECs in optical lattices [12] has greatly stimulated the interest in nonlinear, periodic models. In this case, vortices may appear in the form of self-localized discrete vortex solitons. These have been studied extensively in a variety of different configurations and lattice geometries, not only theoretically [13-21] but also with numerous experimental realizations in optically induced photonic lattices [22-27].

The nonlinear Schrödinger equation has served as the most prominent model in this context, either in its continuous (NLS) or discrete (DNLS) [28] form. For a continuous model, the TC is defined as the phase accumulation when going a full revolution along a (small) closed contour surrounding the phase dislocation. This definition is obviously no longer applicable for a discrete model, but the TC can in a corresponding way be defined as the summation of phase differences between neighboring sites along a closed loop of sites [20].

Even though vortices fundamentally are two-dimensional (2D) objects, one may consider them also in (quasi-)onedimensional (1D) settings, where the sites are arranged in a ring

\footnotetext{
*petej@ifm.liu.se

${ }^{\dagger}$ mjn@ifm.liu.se; https://people.ifm.liu.se/majoh
}

formation. This may serve as a model for, e.g., circular fiber arrays [29], multicore fibers [30,31], and BECs in a necklace potential [32]. Of interest here is also the versatile method to create small photonic lattices that was presented in the recent work by Ref. [27].

In rotationally invariant systems, both angular momentum and TC are conserved, but the introduction of a periodic potential will both break this continuous symmetry and remove the conservation. It is then possible to have a charge flipping vortex (CFV), where the TC is dynamically changing. Charge flipping will in certain cases occur in an irregular and unpredictable way, and is rather associated with the destruction of the vortex [33], but if it can be executed in a controlled manner, then it might be a process which can be utilized in possible applications, e.g., for realizing all-optical switches $[34,35]$.

Of special interest then is that discrete vortices can experience periodic charge flipping, where the TC periodically alternates between a positive and negative value. Periodic charge flipping can be achieved in different ways, e.g., by perturbing a nonflipping vortex configuration, as seen with a single vortex in a square lattice [18], vortices in 1D ringlike lattices $[34,36]$, and multivortex structures in ringlike lattices coupled to a central site [35,37]. It may also occur "spontaneously," as for instance seen for a hexagonal lattice with anisotropic coupling [26], and for vortices with higher TC in a square lattice [24].

The CFVs studied in Refs. [34,36] exist in 1D ringlike lattices with $f=4 l$ sites, $l \in \mathbb{N}^{+}$, and have the simple analytical form

$$
\begin{aligned}
\psi_{2 j-1}(t) & =(-1)^{j} a e^{\left(i a^{2} t+i \alpha\right)}, \\
\psi_{2 j}(t) & =(-1)^{j} b e^{\left(i b^{2} t+i \beta\right)},
\end{aligned}
$$

$j=1, \ldots, 2 l[34,36]$. These solutions effectively split the lattice into two uncoupled and staggered sublattices, consisting of the odd and even sites, respectively. The TC will periodically flip between $\pm l$ with frequency $\left(a^{2}-b^{2}\right)$ [34]. This type of CFV can be obtained by perturbing a constant amplitude 
vortex, $\psi_{n} \propto e^{i \pi n / 2}$ (see Sec. III), with particular linear eigenmodes that have eigenvalue zero [34]. Because of the $\mathrm{U}(1)$ symmetry of the DNLS model, there are always at least two eigenvalues equal to zero, belonging to the so called "phase mode," which only produces an overall phase rotation of the solution [38], but in the case of the $\psi_{n} \propto e^{i \pi n / 2}$ vortex there are two additional zero eigenvalues, which produce the CFV. This is because this vortex actually is a special case of a CFV, with $a=b, \beta-\alpha=\pi / 2$ in Eq. (1) and thus an infinite charge flipping period. Applying this type of perturbation can therefore be considered as moving along a continuous family of CFVs. The existence of CFVs in a four-site lattice was experimentally confirmed in Ref. [27].

It was recently investigated in Ref. [39] whether it would be possible to use a similar strategy to obtain periodic CFVs in a six-site lattice (hexamer), i.e., whether CFVs can be obtained by applying a perturbation of linear eigenmodes to a constant amplitude vortex $\psi_{n} \propto e^{i \theta n}$. It was found that this is indeed the case, but that the magnitude of the perturbation must overcome a (quite large) threshold value. These CFVs do however possess a translational antisymmetry, with $\psi_{n+3}=$ $-\psi_{n}$, which implies that they actually correspond to solutions in the three-site lattice (trimer) with reversed sign on either the coupling or nonlinearity.

The objective of this paper is to give a more detailed description and further explore the properties of CFVs in DNLS trimers with opposite signs (defocusing) and hexamers with the same signs (focusing) on nearest neighbor coupling and nonlinearity. We will show numerically that CFVs exist in continuous families of exact two-frequency quasiperiodic solutions, and that these families will bifurcate with two different stationary solutions: the single depleted well (SDW) and a certain dimerlike solution. We may therefore also determine the minimum energy difference between a constant amplitude vortex and quasiperiodic CFV with the same norm, which may be interpreted as a "charge flipping barrier." The CFVs will, unlike the CFVs in $4 l$-site lattices [34], have periodic oscillations in the site amplitudes. Since they can be calculated with high numerical accuracy, it is also possible to determine their regions of linear stability using Floquet analysis.

The CFVs found in Ref. [39] can be viewed as perturbed quasiperiodic CFVs, resulting essentially in high frequency amplitude oscillations added "on top" of the quasiperiodic CFVs. A deeper understanding of the effect of perturbing constant amplitude vortices, both regarding the results in Ref. [39] and new ones presented in this paper, is therefore obtained in the light of the results for the quasiperiodic CFVs.

The outline of the paper is as follows. In Sec. II we introduce the DNLS model and discuss certain properties of it, before in Sec. II A describing how the linear stability of stationary and (quasi)periodic solutions is determined, and how adequate perturbations are constructed from the linear eigenmodes. In Sec. III certain stationary solutions are reviewed that will be of importance for the rest of the paper: the constant amplitude vortex, the SDW solution, and the dimerlike solution. In Sec. IV we describe how the Newton-Raphson algorithm can be used to find and also follow families of two-frequency quasiperiodic solutions. In Sec. V A we begin by discussing how families of quasiperiodic solutions can be followed from a constant amplitude vortex to a SDW solution, before showing in Sec. V B how exactly quasiperiodic CFVs can be numerically obtained by perturbing the SDW with certain linear eigenmodes. In Sec. V C we will discuss certain properties and display examples of the dynamics of the quasiperiodic CFVs, and it is shown numerically that they exist in continuous families which, below a certain norm, will bifurcate with the SDW solution in one direction and the dimerlike solution in the other. We also discuss the concept of a charge flipping barrier, being the minimal energy difference between a constant amplitude vortex and quasiperiodic CFVs with the same norm. In Sec. VI we will use another strategy to obtain CFVs, which was the one used in Ref. [39], namely to apply a large perturbation, consisting of a linear combination of linear eigenmodes, to a constant amplitude vortex. We will also discuss the effect perturbations on the quasiperiodic CFVs have on the charge flipping barrier. In Sec. VII we determine the linear stability of the quasiperiodic solutions from Sec. V and demonstrate some examples of unstable dynamics, before ending with a summary and conclusions in Sec. VIII.

\section{MODEL}

We will consider a one-dimensional $f$-site discrete nonlinear Schrödinger (DNLS) equation with periodic boundary conditions,

$$
i \dot{\psi}_{n}+C\left(\psi_{n-1}+\psi_{n+1}\right)+\left|\psi_{n}\right|^{2} \psi_{n}=0,
$$

with the dot indicating a derivative with respect to the variable $t$, which we will refer to as time, as it would be for, e.g., BECs in optical lattices. It may however represent another variable, such as propagation length for light in a photonic crystal.

We will consider DNLS trimers with opposite signs of coupling and nonlinearity (defocusing case) and hexamers with coupling and nonlinearity of equal signs (focusing case). We have chosen to alter the sign on the coupling constant $C$, rather than the nonlinearity, with $C$ taken to be only \pm 1 , since the magnitude of the coupling can be changed by rescaling $\psi_{n}$ and $t$ (the choice of sign for the given lattice is discussed later). Note though that this is equivalent to the perhaps more common choice of varying the sign of the nonlinearity while keeping the coupling constant $C$ fixed, since a complex conjugation of $\left\{\psi_{n}\right\}$ together with a sign change of both the coupling constant and nonlinearity leaves the model invariant.

With the set of canonical conjugate variables $\left\{\psi_{n}, i \psi_{n}^{*}\right\}$, Eq. (2) can be derived from the following conserved Hamiltonian:

$$
H\left(\left\{\psi_{n}, i \psi_{n}^{*}\right\}\right)=\sum_{n=1}^{f}\left[-C\left(\psi_{n} \psi_{n+1}^{*}+\psi_{n+1} \psi_{n}^{*}\right)-\frac{1}{2}\left|\psi_{n}\right|^{4}\right] .
$$

Because of the global U(1) symmetry of the model, it also conserves the norm $N=\sum_{n=1}^{f}\left|\psi_{n}\right|^{2}$, being the number of particles in the context of BECs and Poynting power for optical systems.

It is convenient to write certain solutions of the DNLS model as $\psi_{n}(t)=\phi_{n}(t) e^{i \omega_{0} t}$ and study them in a corotating 
frame:

$$
i \dot{\phi}_{n}+C\left(\phi_{n-1}+\phi_{n+1}\right)+\left|\phi_{n}\right|^{2} \phi_{n}-\omega_{0} \phi_{n}=0 .
$$

Important examples include stationary solutions, for which $\left\{\phi_{n}\right\}$ are time independent, and solutions where $\left\{\phi_{n}(t)\right\}$ are periodic and $\left\{\psi_{n}(t)\right\}$ thus generally quasiperiodic in the original frame [38].

Reference [39] considered a hexamer (six-site lattice) with positive coupling. The CFVs that were found in this work all possessed a translational antisymmetry: $\left[\psi_{1}(t), \psi_{2}(t), \psi_{3}(t),-\psi_{1}(t),-\psi_{2}(t),-\psi_{3}(t)\right]$. Solutions of this type can actually be mapped on a corresponding solution in the trimer with negative coupling, $\left[\psi_{1}(t),-\psi_{2}(t), \psi_{3}(t)\right]$, and we can therefore restrict our search for CFVs to this trimer, since it simultaneously gives us CFVs for the hexamer with positive coupling. Note that the hexamer is restricted to a subpart of its phase space by doing this, and that it may very well be possible to find other types of CFVs which break the antisymmetry, but this will not be explored in this paper. It will however be necessary to execute the stability analysis of the hexamer CFVs separately (Sec. VII), since we otherwise would disregard perturbations which break the antisymmetry. Note also that this mapping is different from the trivial mapping where a solution for a smaller lattice is repeated in a larger one, since we now connect two physically different models. The trimer and hexamer can thus be considered as the fundamental (i.e., smallest) lattices where these CFVs exist, for negative and positive coupling (or nonlinearity), respectively, and these two lattices will therefore be the focus of this paper. The norm and Hamiltonian scale linearly with the number of sites, actually for both types of mappings described above, and we will therefore work with Hamiltonian and norm per site, $\bar{N}=N / f$ and $\bar{H}=H / f$, throughout the paper.

From Eq. (4), a continuity equation can be obtained to determine the power flows in the lattice,

$$
\frac{d\left|\phi_{n}\right|^{2}}{d t}=J_{n-1}-J_{n},
$$

where

$$
J_{n}=2 C \operatorname{Im}\left(\phi_{n}^{*} \phi_{n+1}\right)=2 C \sqrt{\left|\phi_{n} \phi_{n+1}\right|} \sin \left(\vartheta_{n+1}-\vartheta_{n}\right)
$$

is the current from site $n$ to site $n+1$, and $\phi_{n}=\left|\phi_{n}\right| \exp \left(i \vartheta_{n}\right)$. Related to this is the topological charge (TC) of the vortex, which is defined as [20]

$$
\mathrm{TC}=\frac{1}{2 \pi} \sum_{n=1}^{f} \arg \left(\psi_{n}^{*} \psi_{n+1}\right),
$$

with $\left|\arg \left(\psi_{n}^{*} \psi_{n+1}\right)\right| \leqslant \pi$, implying that $|\mathrm{TC}| \leqslant f / 2$.

\section{A. Linear stability}

The linear stability of a solution $\left\{\phi_{n}^{(0)}(t)\right\}$ to Eq. (4) is determined by considering the time evolution of an infinitesimal perturbation $\left\{\epsilon_{n}(t)\right\}$. The (linearized) equations of motion for $\epsilon_{n}(t)$ are given by

$$
\begin{aligned}
& \dot{\xi}_{n}=-C\left(\eta_{n+1}+\eta_{n-1}\right)-\left(x_{n}^{2}+3 y_{n}^{2}\right) \eta_{n}-2 x_{n} y_{n} \xi_{n}+\omega_{0} \eta_{n}, \\
& \dot{\eta}_{n}=C\left(\xi_{n+1}+\xi_{n-1}\right)+\left(3 x_{n}^{2}+y_{n}^{2}\right) \xi_{n}+2 x_{n} y_{n} \eta_{n}-\omega_{0} \xi_{n},
\end{aligned}
$$

where the solution and perturbation have been decomposed into their real and imaginary parts: $\phi_{n}^{(0)}(t)=x_{n}(t)+i y_{n}(t)$, $\epsilon_{n}(t)=\xi_{n}(t)+i \eta_{n}(t)$.

For a stationary solution, $x_{n}$ and $y_{n}$ are constant in time, and we may therefore express the equations above through the (time-independent) matrix relationship

$$
\left(\begin{array}{l}
\left\{\dot{\xi}_{n}(t)\right\} \\
\left\{\dot{\eta}_{n}(t)\right\}
\end{array}\right)=F\left(\begin{array}{l}
\left\{\xi_{n}(t)\right\} \\
\left\{\eta_{n}(t)\right\}
\end{array}\right),
$$

with $F$ being the functional (or Jacobian) matrix of $\left\{\dot{x}_{n}, \dot{y}_{n}\right\}$ evaluated at the stationary point. $F$ is an infinitesimally symplectic matrix [40], so if $\lambda$ is an eigenvalue, then so are also $\lambda^{*},-\lambda$, and $-\lambda^{*}$. The stationary solution is linearly stable if none of the eigenvalues of $F$ have a positive real part, which implies that all eigenvalues must be found on the imaginary axis.

Should $\left\{\phi_{n}^{(0)}(t)\right\}$ instead be a periodic solution, then the linear stability may be studied using Floquet analysis, where the evolution of $\left\{\epsilon_{n}(t)\right\}$ is considered only after multiples of the period $T$. The linear stability is determined by analyzing the eigenvalues of the $2 f \times 2 f$ dimensional Floquet matrix, given by the mapping

$$
\left(\begin{array}{l}
\left\{\xi_{n}(T)\right\} \\
\left\{\eta_{n}(T)\right\}
\end{array}\right)=T_{0}\left(\begin{array}{l}
\left\{\xi_{n}(0)\right\} \\
\left\{\eta_{n}(0)\right\}
\end{array}\right),
$$

which is obtained by integrating Eq. (8). $T_{0}$ is now a symplectic matrix [40], implying that if $\lambda$ is one of its eigenvalues, then so are also $\lambda^{*}, 1 / \lambda$, and $1 / \lambda^{*}$. A periodic solution is linearly stable if none of the Floquet matrix's eigenvalue moduli exceed 1 , which implies that all eigenvalues must reside on the unit circle.

For a pair of complex eigenvalues $\lambda$ and $\lambda^{*}$, the corresponding eigenmodes $\left\{\xi_{n}^{(\lambda)}, \eta_{n}^{(\lambda)}\right\}$ and $\left\{\xi_{n}^{\left(\lambda^{*}\right)}, \eta_{n}^{\left(\lambda^{*}\right)}\right\}$ can be chosen as complex conjugates of each other (i.e., $\xi_{n}$ and $\eta_{n}$ are complex for both of them), and to create an adequate perturbation, $\left\{p_{n}\right\}$, it is necessary to take a linear combination of the form

$$
\begin{aligned}
\left\{\operatorname{Re}\left(p_{n}\right), \operatorname{Im}\left(p_{n}\right)\right\}= & C_{1}\left(\left\{\xi_{n}^{(\lambda)}+\xi_{n}^{\left(\lambda^{*}\right)}, \eta_{n}^{(\lambda)}+\eta_{n}^{\left(\lambda^{*}\right)}\right\}\right) \\
& +i C_{2}\left(\left\{\xi_{n}^{(\lambda)}-\xi_{n}^{\left(\lambda^{*}\right)}, \eta_{n}^{(\lambda)}-\eta_{n}^{\left(\lambda^{*}\right)}\right\}\right),
\end{aligned}
$$

with $C_{1}, C_{2} \in \mathbb{R}$.

As a family of solutions is followed for a continuous parameter, the stability eigenvalues will also change continuously. If the family is followed in a regime where the solutions are stable, then pairs of Floquet eigenvalues will rotate around the unit circle, one eigenvalue in each direction. For quasiperiodic solutions of the type $\psi_{n}(t)=\phi_{n}(t) e^{i \omega_{Q P} t}, \phi_{n}(t+T)=\phi_{n}(t)$, there will however be at least two pairs of eigenvalues that always reside in +1 . One pair is due to the $U(1)$ symmetry of the DNLS model, and thus present for all solutions, belonging to the phase mode, which only produces an overall phase rotation of the solution, and the other pair belongs to the eigenmode $\epsilon_{n}=\dot{\phi}_{n}$. For the trimer, this means that there is only one pair of eigenvalues left that can rotate around the unit circle, and instabilities can therefore only arise if this pair collide either in -1 or +1 , and thereafter goes out along the real axis. For the hexamer there is also the possibility that two pairs of eigenvalues collide at $e^{ \pm i \alpha}, \alpha \neq 0, \pi$, and go away from the unit circle into the complex plane (not along the real axis), resulting in an oscillatory instability [41]. It is possible 

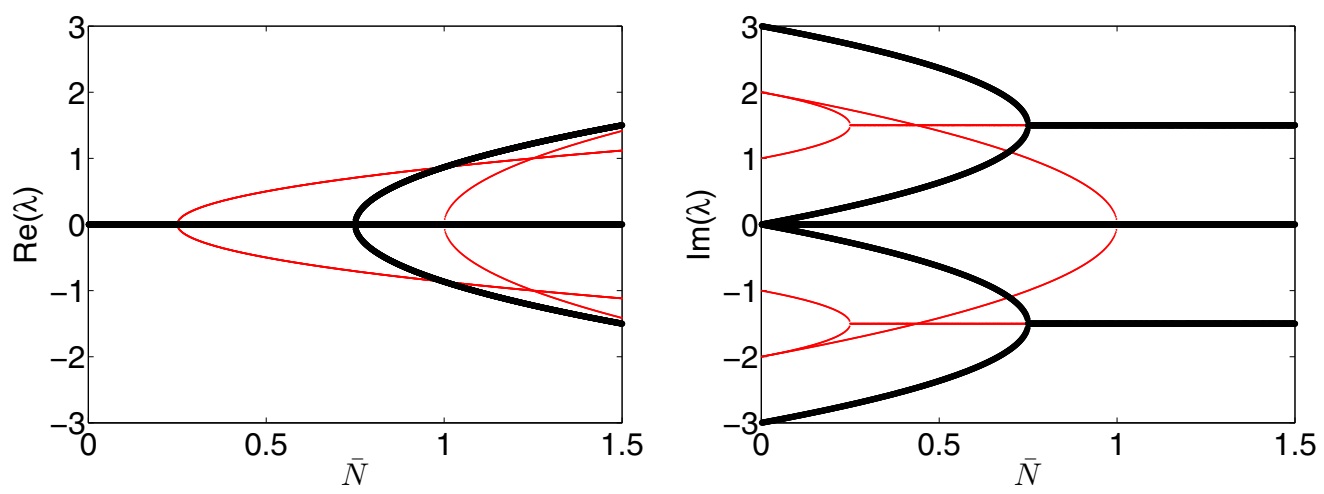

FIG. 1. (Color online) Stability of the $\kappa= \pm 1$ constant amplitude vortex for both trimer $(C=-1)$ and hexamer $(C=+1)$. Left (right) figure shows the real (imaginary) part of the eigenvalues of the functional matrix Eq. (9) as a function of the norm per site. The thick, black lines belong to both the trimer and hexamer, while the thinner, red lines only belong to the hexamer.

that when two eigenvalues collide on the unit circle, they will only go away from the unit circle very briefly, i.e., over a small parameter range, and also only a short distance, thereby resulting in a very weak instability.

\section{STATIONARY SOLUTIONS}

In this section we will briefly review some stationary solutions of the DNLS model which are of relevance to the CFVs. These solutions have been studied more thoroughly in other papers, and the reader is directed to these for further details.

The DNLS equation Eq. (2) supports constant amplitude vortices, $\psi_{n}(t)=\psi_{0} e^{i(\omega t+\theta n)}[42,43]$. In a finite, periodic lattice the (transverse) wavelength becomes quantized, $\theta_{\kappa}=$ $2 \pi \kappa / f$, with $\kappa=0, \pm 1, \ldots, \pm(f / 2-1), f / 2$ ( $f$ even) or $\kappa=0, \pm 1, \ldots, \pm(f-1) / 2$ ( $f$ odd $)$. The frequency of the vortices in the original frame is given by the dispersion relation

$$
\omega_{V}=\left|\psi_{0}\right|^{2}+2 C \cos \left(\theta_{\kappa}\right)=\bar{N}+2 C \cos \left(\theta_{\kappa}\right) .
$$

Reference [39] obtained CFVs by perturbing a $\kappa=1$ vortex in the hexamer with positive coupling. This vortex possesses the above mentioned antisymmetry, $\psi_{n}=-\psi_{n+3}$, and will correspond to a $\kappa=-1$ vortex in the trimer with negative coupling. In Fig. 1, the real and imaginary parts of the eigenvalues of the functional matrix Eq. (9) are plotted as a function of the norm per site. The trimer vortex with $C=-1$ is linearly stable for $\bar{N}<0.75$, while the hexamer vortex with $C=+1$ is linearly stable for $\bar{N}<0.25$.

Since changing the sign of the coupling together with a staggering transformation leaves the model invariant, all results for $\kappa= \pm 1$ hexamer vortices become transferable to $\kappa= \pm 2$ vortices in the hexamer with opposite sign of the coupling, and vice versa. We may therefore restrict our attention to one type of coupling constant $C$ for hexamers, chosen to be positive. Note also that a $\kappa= \pm 2$ vortex essentially is two $\kappa= \pm 1$ trimer vortices repeated in the lattice (the "trivial mapping" discussed in Sec. II), and that any CFV obtained by perturbing a $\kappa= \pm 1$ trimer vortex should therefore correspond to a CFV in the hexamer (with same sign on the coupling) obtained by perturbing a $\kappa= \pm 2$ vortex. Since no CFVs were found in Ref. [39] by perturbing $\kappa= \pm 2$ vortices for the hexamer with positive coupling, we will not look for CFVs in the trimer with positive coupling (where the stationary vortex is unconditionally linearly stable $[42,43])$.

The two other stationary solutions that we will discuss are, unlike the vortex, non-current-carrying, and can be expressed as real solutions to Eq. (4) in a corotating frame. The first one is the so-called "single depleted well" (SDW) solution [44]. It has the form $\psi_{n}=(3 \bar{N} / 2)^{1 / 2}[1,-1,0] e^{i \omega_{\text {SDW }} t}$, or correspondingly $(3 \bar{N} / 2)^{1 / 2}[1,1,0,-1,-1,0] e^{i \omega_{\mathrm{SDw}} t}$, which plugged into Eq. (2), with $C=-1$ for trimer and $C=+1$ for hexamer, gives the


FIG. 2. (Color online) Same as Fig. 1 but for the "single depleted well" (SDW) solution $(3 \bar{N} / 2)^{1 / 2}[1,-1,0] e^{i \omega_{\text {SDW }} t}$ and corresponding hexamer solution. The eigenvalues displayed are either real, imaginary, or zero and CFVs can be obtained from the SDW by perturbing it in a proper way (see text for details) with the eigenmodes that exist for both trimer and hexamer and have real eigenvalues. 

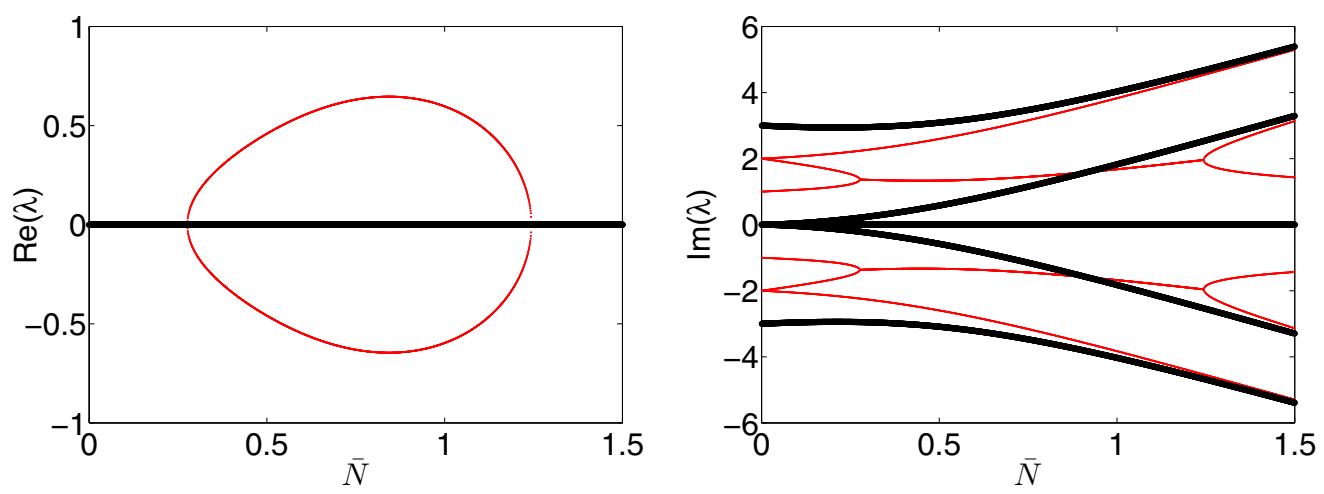

FIG. 3. (Color online) Same as Fig. 1 but for the dimerlike solution $[A,-B,-B] e^{i \omega_{D L} t}$ and the corresponding hexamer solution. CFVs can be obtained from the dimerlike solution by perturbing it with the eigenmodes, which for the trimer have the smallest (in magnitude) imaginary eigenvalues.

dispersion relation

$$
\omega_{\mathrm{SDW}}=1+\frac{3 \bar{N}}{2} .
$$

In Fig. 2 we examine the stability of the SDW solution, which indicates that this solution always is unstable [45]. All the eigenvalues of the functional matrix are, both for the trimer and hexamer, either real, imaginary, or zero.

The second non-current-carrying solution is one of the "dimerlike configurations" studied in Ref. [44] (the $A_{3}$ solution) and has the form $\psi_{n}=[A,-B,-B] e^{i \omega_{D L} t}$ for the trimer with $C=-1$, or correspondingly $[A, B,-B,-A,-B, B] e^{i \omega_{D L} t}$ for the hexamer with $C=+1$, with $A, B \in \mathbb{R}^{+}$and $A>B$. $A$ and $B$ can be obtained in a parametrized form; see either Ref. [44] or Ref. [45] where the corresponding hexamer solution is discussed (the $[\uparrow . . \downarrow .$.$] solution). This solution approaches \left[(3 \bar{N})^{1 / 2}, 0,0\right]$ $\left(\left[(3 \bar{N})^{1 / 2}, 0,0,-(3 \bar{N})^{1 / 2}, 0,0\right]\right)$ asymptotically as the norm $N \rightarrow \infty$. In Fig. 3 we display the stability eigenvalues of the dimerlike solution. The eigenvalues belonging to both the trimer and hexamer are either imaginary or zero, and the trimer is thus always stable, while the hexamer has four complex eigenvalues in the region $0.276<\bar{N}<1.244$, where it thus becomes unstable. In Fig. 4 the Hamiltonian per site for both the SDW and dimerlike solution, together with some additional solutions that we will encounter later, are plotted relative to the Hamiltonian per site of the $\kappa= \pm 1$ vortex.

\section{METHOD FOR FINDING QUASIPERIODIC SOLUTIONS}

In this section we will describe the method used to find and follow families of exact quasiperiodic solutions. Quasiperiodic solutions of the type $\psi_{n}(t)=\phi_{n}(t) e^{i \omega_{Q P} t}$, with $\left\{\phi_{n}(t+T)\right\}=$ $\left\{\phi_{n}(t)\right\}$, become periodic with period $T$ in a frame rotating with $\omega_{Q P}$. One can therefore search for them using a NewtonRaphson (NR) algorithm that solves $\mathcal{F}\left(\left\{\phi_{n}(0)\right\}\right) \equiv\left\{\phi_{n}(T)\right\}-$ $\left\{\phi_{n}(0)\right\}=0$, where $\left\{\phi_{n}(T)\right\}$ is obtained by integrating Eq. (4) with $\omega_{0}=\omega_{Q P}$. These solutions belong to two-parameter families, meaning that two parameters of the sought-after solution must be specified in the NR algorithm, e.g., the frequency of the rotating frame $\omega_{Q P}$, the time period $T$ in the rotating frame, and/or the norm $N$. The norm is in practice specified by adding an extra equation and using the NR algorithm to solve

$$
\mathcal{F}\left(\left\{\phi_{n}(0)\right\}, \omega_{Q P}\right)=\left\{\begin{array}{l}
\left\{\phi_{n}(T)\right\}-\left\{\phi_{n}(0)\right\}=0, \\
\sum_{n=1}^{f}\left|\phi_{n}\right|^{2}-N=0,
\end{array}\right.
$$

where $N$ is the desired norm of the solution. We now also treat $\omega_{Q P}$ as a variable in the algorithm. One could equally well have used $T$ instead of $\omega_{Q P}$, but this will not be done in this paper. The NR algorithm is iterated until a chosen convergence criterion is fulfilled, $\|\mathcal{F}\|<\delta$ ( $l_{2}$ norm), with $\delta$ taken to be $10^{-12}$ in this paper.

In the execution of the NR algorithm, one tries to invert the matrix $\left(I-T_{0}\right)$, where $I$ is the identity matrix and $T_{0}$ is the Floquet matrix given by Eq. (10) [13]. For this type of quasiperiodic solutions, the Floquet matrix has two pairs

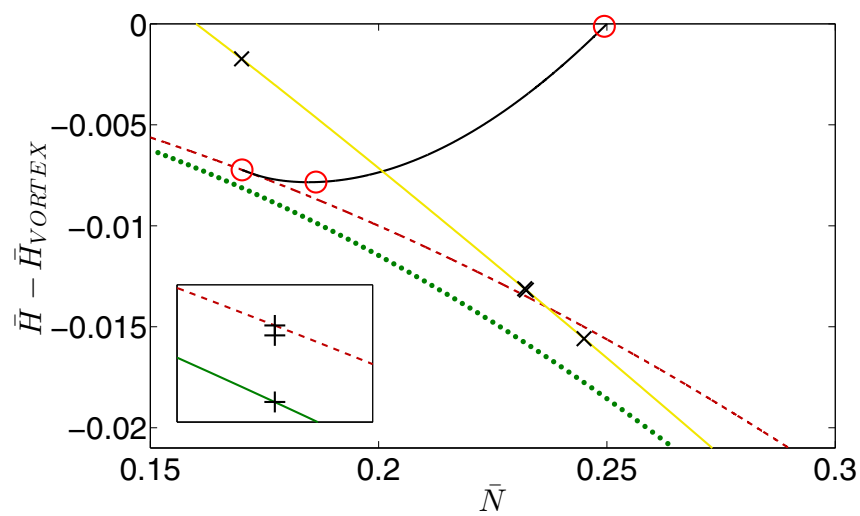

FIG. 4. (Color online) The Hamiltonian per site of different solutions, relative to the Hamiltonian per site of the $\kappa= \pm 1$ constant amplitude vortex, as a function of norm per site. The lines plotted belong to the SDW (dashed red), the dimerlike (dotted green), the quasiperiodic family discussed in Sec. V A (solid black), and the $\kappa= \pm 1$ constant amplitude vortex perturbed with eigenmodes (solid yellow [gray]). The quasiperiodic solutions shown in Fig. 5 are marked as red circles, while the perturbed vortices shown in Fig. 7 are marked as black crosses [note that the crosses for Fig. 7(b) and Fig. 7(c) lie very close to each other]. The inset zooms in on the region plotted in Fig. 6, with the three CFV solutions located at $\bar{N}=1 / 6$, $\bar{H}-\bar{H}_{\text {VORTEX }}=-6.94 \times 10^{-3},-7.05 \times 10^{-3},-7.78 \times 10^{-3}$. 
of eigenvalues equal to unity (see Sec. II A), and the matrix ( $I-T_{0}$ ) is thus noninvertible. One way to resolve this would be to restrict the search to time-reversible solutions, but since we are interested in current-carrying solutions, we cannot do this. Note though that the CFVs themselves might actually be time reversible (since the current is reversed), but that the constant amplitude vortices, and the quasiperiodic families that bifurcate from them, surely are not. We will instead use the pseudoinverse of $\left(I-T_{0}\right)$, calculated using the singular value decomposition [13].

By recursively iterating the NR algorithm, we can follow continuous one-parameter subfamilies of these quasiperiodic solutions. This means that we will use a quasiperiodic solution that has been calculated with the NR algorithm as the initial guess in a new round with the NR algorithm, but this time with slightly shifted values of the specified parameters (i.e., $\omega_{Q P}, T$, and/or $\left.N\right)$. Typically one follows a family defined by a fixed value of one of the parameters, thereby only changing the other, but it is in principle possible to follow any curve in parameter space.

When going through this procedure, it is necessary to have a good starting guess to begin with. This can be obtained by perturbing stationary solutions with linear eigenmodes of the functional matrix (9). For stable eigenmodes, a linear combination must be taken according to Eq. (11). The natural choice would in this case be to set (either as specified parameters or initial guesses) $\omega_{Q P}$ to that of the stationary solution and $T=2 \pi /|\lambda|$, with $|\lambda|$ being the modulus of the associated eigenvalue. It is however generally necessary to tweak the value of these parameters slightly for the NR algorithm to converge, since generally only an infinitesimal perturbation will oscillate with exactly $2 \pi /|\lambda|$.

It is actually possible in some special cases to also obtain quasiperiodic solutions by perturbing the stationary solution with unstable eigenmodes, as we will see an example of in Sec. V B. It is however no longer possible to use $T=2 \pi /|\lambda|$, but initial guesses for $\omega_{Q P}$ and $T$ can instead be determined by graphical means.

\section{CHARGE FLIPPING VORTICES}

\section{A. Non-charge-flipping quasiperiodic family between constant amplitude vortex and SDW}

By using a $\kappa= \pm 1$ constant amplitude vortex perturbed with a linear combination [according to Eq. (11)] of the two eigenmodes with the smallest imaginary eigenvalues as the initial guess in the NR algorithm, it is possible to follow families of (non-charge-flipping) quasiperiodic solutions. The existence of these families has been rigorously proven in Ref. [46]. In Fig. 5 we show $\left|\psi_{n}(t)\right|^{2}$ for some quasiperiodic solutions that belong to a one-parameter subfamily with $\omega_{Q P}=1.25$ which bifurcates from the $\kappa=-1(\kappa=1$ for the hexamer) vortex with $\bar{N}=0.25$, which have been followed for increasing $T$.

As $T$ is increased, the solutions in this family approach a part of phase space where a hole (empty site) is "tunneling" through the lattice. By looking at $\psi_{n}(t)$, one sees that when one site is nearly empty, the other two are close to being the negative of each other. The trajectory of the solution displayed
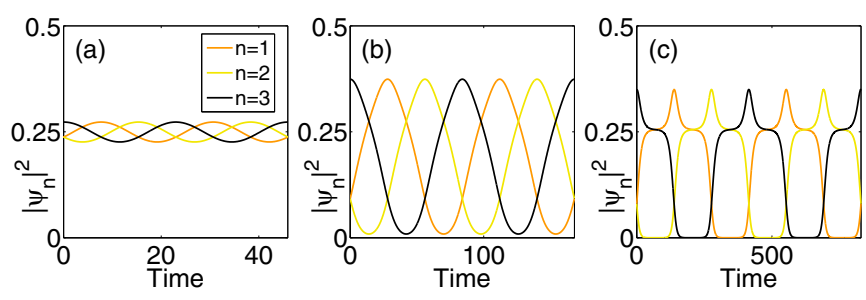

FIG. 5. (Color online) Time evolution of $\left|\psi_{n}(t)\right|^{2}$ for some quasiperiodic solutions belonging to a family with fixed $\omega_{Q P}=1.25$ that bifurcates with the $\bar{N}=0.25, \kappa=-1$ constant amplitude vortex in the $C=-1$ trimer $(\kappa=+1$ constant amplitude vortex in the $C=+1$ hexamer). The locations of these solutions are marked with red circles in the norm-Hamiltonian diagram Fig. 4. Panel (a) is close to the $\kappa= \pm 1$ constant amplitude vortex while panel (c) is close to the stationary SDW solution.

in Fig. 5(c) is thus close in phase space to the SDW solution discussed in Sec. III, which also can be seen in Fig. 4 where $\bar{H}$ of this quasiperiodic family clearly approaches $\bar{H}$ of the SDW solution. The position of this bifurcation point can be calculated by setting $\omega_{Q P}=\omega_{\mathrm{SDW}}$, giving $\bar{N}=1 / 6$ for this particular family, which is in agreement with Fig. 4.

We thus have two-parameter families of quasiperiodic solutions which in one direction bifurcate with a $\kappa= \pm 1$ constant amplitude vortex and in the other direction bifurcate (when $T \rightarrow \infty$ ) with a SDW solution. Let us just emphasize that it is possible to follow the families along many different paths (i.e., one-parameter subfamilies) from the constant amplitude vortices to the SDWs, and that these may be for decreasing, increasing, or fixed norm.

Being able to follow these families all the way from the vortex to the SDW is however only valid below a certain norm. If a family is followed for fixed norm $\bar{N} \gtrsim 0.48$ for increasing time period from the constant amplitude vortex, then it will bifurcate with the quasiperiodic family that can be followed (for decreasing time period) by perturbing the constant amplitude vortex with the other stable eigenmodes, associated with the larger eigenvalues. At $\bar{N} \approx 0.48$, the eigenvalues $\left|\lambda_{1}\right| \approx 4\left|\lambda_{2}\right|$, and the bifurcation is in fact of a period-quadrupling type. The family can thus be followed for increasing time period in a "loop" where it returns to the constant amplitude vortex, above the norm threshold.

The value of the norm threshold will obviously depend on which one-parameter subfamily is actually followed. It will, e.g., be possible to follow a family from a $\kappa= \pm 1$ constant amplitude vortex with $\bar{N}>0.48$ all the way to the SDW if this is done along a path with decreasing norm. The threshold value $\bar{N} \approx 0.48$ is thus for a family with fixed norm. The linear stability of these quasiperiodic solutions is discussed in Sec. VII.

\section{B. Generating CFVs from the SDW}

CFVs can be obtained by making certain perturbations of the SDW solution. Looking back at Fig. 2, the SDW has two nonzero real eigenvalues that belong to both the trimer and hexamer (the real eigenvalues that belong only to the hexamer will not result in a CFV). The corresponding eigenmodes of the functional matrix, which we label $\left\{\xi_{n}^{+}, \eta_{n}^{+}\right\}$and $\left\{\xi_{n}^{-}, \eta_{n}^{-}\right\}$ 
for the positive and negative eigenvalue, respectively, can be chosen so that $\xi_{n}^{+}=\xi_{n}^{-}$and $\eta_{n}^{+}=-\eta_{n}^{-}$(which we will assume from now). The associated stable and unstable manifolds will correspond to a situation where the SDW's hole, in an infinite time, is "tunneled" exactly one site. Note though that when applying a perturbation consisting of the stable eigenmode, one will only see the last part of the tunneling process, as the hole reaches its destination.

When a perturbation that is constructed by an eigenmode, i.e., $\epsilon_{n}^{ \pm}=\xi_{n}^{+} \pm i \eta_{n}^{+}$, is applied to the SDW solution, it will increase the amplitude on one of the populated sites (pair of sites for the hexamer), while reducing it on the other, so that, e.g., $\left|\psi_{n}+\epsilon_{n}^{ \pm}\right|^{2}=\left[N / 2+\delta_{1}, N / 2-\delta_{2}, \delta_{3}\right]$, with $\delta_{1,2,3} \in \mathbb{R}^{+}$, for the trimer. Note that $\epsilon^{+}$and $\epsilon^{-}$increase and reduce the amplitudes on the same sites. The manifold that is associated with the applied perturbation will tunnel the hole between the site where the hole is located (site 3 in the above example) and the site on which the amplitude is reduced (site 2 in the example). Note also that there is only one stable and unstable eigenmode for each trimer SDW, so changing the sign by which $\epsilon_{n}^{+}$or $\epsilon_{n}^{-}$is applied will change the direction of the tunneling; i.e., $\left|\psi_{n}-\epsilon_{n}^{ \pm}\right|^{2}=\left[N / 2-\delta_{2}, N / 2+\delta_{1}, \delta_{3}\right]$ will lead to tunneling between site 1 and site 3 . This behavior can be compared with the familiar example of the upwards pointing, unstable pendulum [47].

We can intuitively think of the unstable perturbation as pushing the hole away towards another site, while the stable perturbation pulls the hole back. And just as with the pendulum, some linear combinations of the stable and unstable eigenmodes will lead to a hole that is oscillating between only two sites (analogous to librational motion for the pendulum), corresponding to a CFV, while other linear combinations will lead to a hole that is jumping between all three sites (analogous to rotational motion), corresponding to a quasiperiodic solution similar to the one in Fig. 5(c).

To produce a $\mathrm{CFV}$, we expect that we should add the unstable and stable perturbation in such a way so that they, if added separately, would decrease the amplitude on the same site. We can thus create a $\mathrm{CFV}$ by applying a perturbation $\propto\left\{\cos (\gamma) \epsilon_{n}^{+}+\sin (\gamma) \epsilon_{n}^{-}\right\}$for $0<\gamma<\pi / 2$ and $\pi<\gamma<3 \pi / 2$, the two regions of $\gamma$ leading to the hole tunneling in different directions. Note especially that the perturbation $\left\{ \pm \operatorname{Re}\left(\epsilon^{ \pm}\right)\right\}$gives a CFV. This can, since the SDW is real, be thought of as a perturbation only in position, and since a corresponding perturbation of the unstable pendulum leads to librational motion, we can see that the pendulum analogy is consistent. The other parameter intervals (and especially $\left\{ \pm \operatorname{Im}\left(\epsilon^{ \pm}\right)\right\}$) yield the type of quasiperiodic solution discussed in Sec. V A and shown in Fig. 5(c). A purely imaginary perturbation will correspond to a perturbation of the momentum of the pendulum, leading to rotational motion, which also is consistent with the analogy.

\section{The quasiperiodic CFV family}

The NR procedure described in Sec. IV can be used to follow continuous families of exact quasiperiodic CFVs, and we may now use a "properly" perturbed SDW solution (in the sense discussed above) as the starting guess. Note however that this is an unstable perturbation, and that initial guesses for
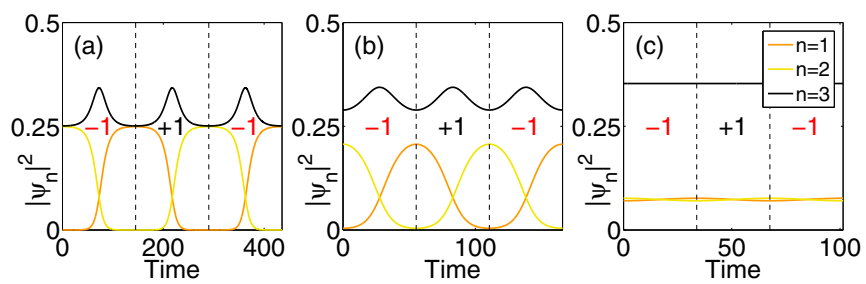

FIG. 6. (Color online) Quasiperiodic CFVs with $\bar{N}=0.1667$. Panel (a) is obtained by using a perturbed SDW as an initial guess in the Newton-Raphson algorithm. Panels (b) and (c) show members of the CFV family that can be followed for decreasing time period. Panel (c) is close to the dimerlike solution, and can be obtained by perturbing it with the linear eigenmodes that have the smallest imaginary eigenvalues. The figures also indicate the value of the TC.

$\omega_{Q P}$ and $T$ therefore are determined graphically. In Fig. 6 we show some solutions of a CFV family, this time with the norm per site fixed to $\bar{N}=1 / 6$ and with $\omega_{Q P}$ treated as a variable in the NR algorithm. As the family is followed for decreasing time period $T$, it will eventually bifurcate with the dimerlike solution, discussed in Sec. III. CFVs [e.g., Fig. 6(c)] can thus also be obtained by perturbing the dimerlike solution, this time with the pair of stable eigenmodes that are associated with the imaginary eigenvalues which go to zero with the norm (see Fig. 3). The frequency of the CFVs will, in a frame rotating with $\omega_{D L}$, approach $2 \pi /|\lambda|, \lambda$ being one of these eigenvalues, as a family of CFVs is followed for decreasing time period towards a dimerlike solution.

It will however only be possible to follow continuous families of CFVs all the way from the SDW to the dimerlike solution below a certain norm. For sufficiently large $\bar{N}$, quasiperiodic CFVs will not bifurcate from the SDW solution. It is difficult to determine exactly when the CFVs stop bifurcating with the SDW, both because the time period is going towards infinity, and because the convergence rate of the NR algorithm slows down, as a bifurcation point is approached. We can however say that up to $\bar{N} \approx 0.6$, the quasiperiodic CFVs can be followed to a solution very close to the SDW. Around this $\bar{N}$, the CFVs start to bifurcate with non-charge-flipping quasiperiodic solutions, as the time period is increased. As $\bar{N}$ is increased, this bifurcation happens further and further away from the SDW, but it is possible to follow the family of CFVs to a solution which is quite close to the SDW, up to around $\bar{N} \approx 0.8$.

This bifurcation with non-charge-flipping solutions is also noticed when one uses the method described in Sec. V B to generate CFVs by perturbing an SDW. At around $\bar{N} \gtrsim 0.6$ the dynamics obtained when adding a perturbation will not be as simple as in Fig. 6(a), but it will also display additional oscillations in the amplitude. This effect is further enhanced as $\bar{N}$ is increased, and it will eventually be necessary to add quite a substantial perturbation for the NR algorithm to converge to a quasiperiodic solution, resulting in a solution far from the SDW.

In analogy with the quasiperiodic family discussed in Sec. VA, the family of CFVs that bifurcates from the dimerlike solution will, when followed for fixed norm $\bar{N} \gtrsim 1.12$ and increasing time period, bifurcate with the family of quasiperiodic solutions that is obtained by perturbing the 
dimerlike solution with the other stable eigenmodes. It is around $\bar{N} \approx 1.12$ that the ratio between the two eigenvalues is approximately 2 (see Fig. 3), and the bifurcation between these two families is indeed of a period doubling type. Note that the constant amplitude vortex is unstable, even for the trimer, for such large values of $\bar{N}$.

The exact quasiperiodic CFVs are thus (below the norm threshold) trapped between two stationary solutions: the SDW and the dimerlike. Figure 4 therefore shows where these CFVs exist in a norm/Hamiltonian diagram. From Fig. 6 we see that the CFVs consist of one site (two sites for hexamer) which always is populated with at least half of the total norm, and that there is a periodic amplitude inversion between the two other sites (two pairs of sites for the hexamer). This is unlike the CFVs in the $4 l$-site lattices, where the amplitude is time independent on all sites [34]. It is when the mostly populated site gets its minimal value that the TC is flipped. The phase difference between the mostly occupied site and the two others is (for the trimer) equal to $\pi$ at this point, and one may therefore, at least close to the SDW solution, approximate the initial conditions of a CFV with $[\sqrt{N / 2}+\delta,-\sqrt{N / 2}+\delta,-\delta]$, with $\delta$ real and small.

From Fig. 4 it is evident that the smallest energy difference between a $\kappa= \pm 1$ constant amplitude vortex and an exact quasiperiodic $\mathrm{CFV}$ with the same norm equals the energy difference between the $\kappa= \pm 1$ constant amplitude vortex and the SDW solution; i.e.,

$$
\Delta \bar{H}_{\text {barrier }}=-\frac{\bar{N}^{2}}{4} .
$$

This can therefore be viewed as a charge flipping barrier, i.e., the minimum energy it takes to flip the TC while keeping the norm constant, analogous to the Peierls-Nabarro barrier for the translational motion of discrete breathers [48,49]. We must however be careful in how we interpret the barrier, as we will see in Sec. VI when discussing the effect of perturbations on the exact CFVs.

\section{GENERATING CFVs BY PERTURBING VORTICES}

We now turn to a different strategy for generating CFVs, which was the one utilized in Ref. [39], namely to add a large perturbation consisting of certain eigenmodes of the functional matrix to a $\kappa= \pm 1$ constant amplitude vortex. It will not be possible, in contrast to $4 l$-site lattices [34], to generate CFVs by perturbing the $\kappa= \pm 1$ constant amplitude vortex with eigenmodes that have eigenvalues equal to zero, since it is now only the phase mode which has that. We will instead apply the same type of perturbation that was used to create the initial guess for obtaining the quasiperiodic families discussed in Sec. V A, i.e., a linear combination consisting of the two eigenmodes with smallest, imaginary eigenvalues.

In Fig. 7 we display the dynamics of $\left|\psi_{n}^{p}(t)\right|^{2}$, with $\psi_{n}^{p}(0)=$ $\left(0.4+p_{n}\right) e^{-i 2 \pi n / 3}$ for the trimer, for different magnitudes of the perturbation $p_{n}$. The perturbation $p_{n}$ is chosen with $C_{2}=0$ in Eq. (11), the eigenmodes being those of the $\bar{N}=$ $0.16, \kappa= \pm 1$ constant amplitude vortex with $\lambda \approx \pm 0.17 i$ (cf. Fig. 1), so that $p_{n} / \sqrt{\sum_{m=1}^{3}\left|p_{m}\right|^{2}} \approx[-0.3054-0.4692 i$, $-0.3054+0.4692 i, 0.6108]$ for the trimer. Note that the norm
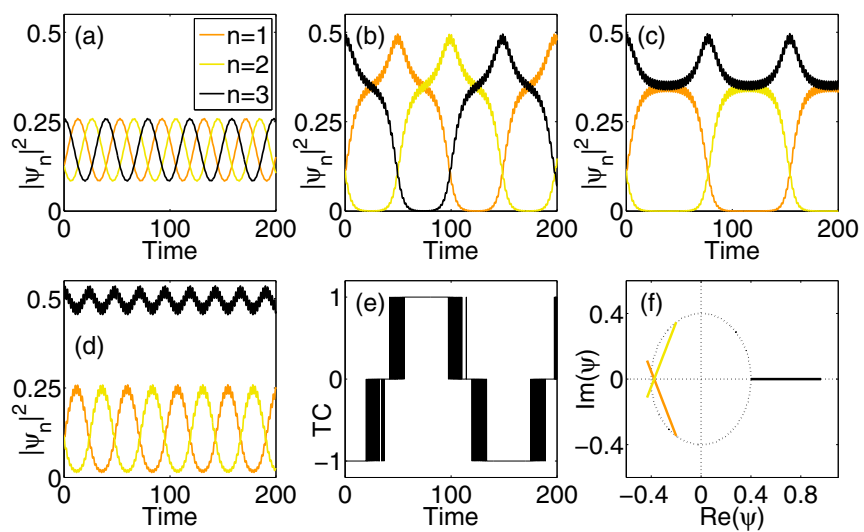

FIG. 7. (Color online) Dynamics of $\left|\psi_{n}\right|^{2}$ for a perturbed $\kappa= \pm 1$ vortex, with norm per site of the perturbation $\sum\left|p_{n}\right|^{2} / f=$ (a) 0.01 , (b) 0.072 , (c) 0.072333 , (d) 0.085 . The TC is constant -1 in panel (a) and almost constant -1 for panel (b) (TC is not shown for these plots), with spikes to 0 for panel (b) during the times when one of the sites has an amplitude close to zero. In panel (c) we have just crossed over to a charge flipping dynamics [cf. Fig. 6(a)], with the associated TC shown in panel (e). Note the fluctuations in TC as it changes between +1 and -1 , due to the high frequency oscillations in the amplitude. Panel (d) shows the dynamics for an even larger perturbation, where we are in between the SDW and dimerlike solution [cf. Fig. 6(b)]. In panel (f), $\psi_{n}(0)$ is shown for increasing magnitude of the perturbation. The locations of the solutions in panels (a)-(d) are marked with black crosses in Fig. 4.

is increasing while the Hamiltonian is decreasing with the magnitude of the perturbation (Fig. 4).

When the perturbation becomes larger than a threshold value [Fig. 7(c)], the dynamics changes to a periodically charge flipping type [39], with the TC associated with Fig. 7(c) shown in Fig. 7(e). The perturbation threshold is in contrast to the CFVs in $4 l$-site lattices, where charge flipping occurs even for infinitesimal perturbations [34]. Figure 7(d) shows the dynamics for an even larger perturbation and Fig. 7(f) displays how $\psi_{n}^{p}(0)$ change as the magnitude of the perturbation increases.

The TC is constant $-1(+1$ for the corresponding hexamer solution) for Fig. 7(a), and has a few spikes to $\mathrm{TC}=0$ for Fig. 7(b) because of the high frequency "noise." The perturbation must be quite strong, so that the periodic charge flipping regime [Fig. 7(c)] almost has been entered, before the conservation of TC is broken [note how close the two crosses for Figs. 7(b) and 7(c) are to each other in Fig. 4]. The TC for the $\kappa= \pm 1$ constant amplitude vortices is thus less sensitive to perturbation in trimer and hexamer lattices, compared with the $4 l$ site lattices.

There is a clear qualitative similarity between Figs. 6 and 7, and the analysis of the dynamics in Fig. 7 is easily done in light of the results for the exactly quasiperiodic CFVs from the previous section. The main difference between the two figures is that the solutions obtained in this section have these high frequency oscillations in the amplitude, which may be considered as a result of perturbations of the exact quasiperiodic CFVs. There is similarly a crossover between Figs. 7(b) and 7(c) [cf. Figs. 5(c) and 6(a)] to the charge flipping regime, which is associated to the SDW solution and can be 
interpreted as a hole which changes from rotating around the whole lattice to oscillating between only two sites.

Figure 7(f) shows $\psi_{n}(0)$ for different magnitudes of the perturbation and it is evident from this figure that the effect of the perturbation is to increase the amplitude on site 3 , but not changing its phase, while at first decreasing the amplitude on site 1 and 2 and drawing their phases closer to each other, until an intersection point is reached, after which their amplitudes are increased and their phases deviate from each other. When the perturbation is increased, and the $\mathrm{CFV}$ region has been entered, the oscillations in $\left|\psi_{n}\right|^{2}$ (not the high frequency oscillations) will therefore at first decrease [cf. Fig. 7(c) and Fig. 7(d)], which is consistent with how the exact quasiperiodic CFVs in Sec. V C change when they approach the dimerlike solution. The intersection point between $\psi_{1}(0)$ and $\psi_{2}(0)$ in Fig. 7(f) is for a perturbation strength of $\sum\left|p_{n}\right|^{2} / f \approx 0.16$, and corresponds to when it is closest to the dimerlike solution. Note though that these are only approximate quasiperiodic solutions, and that the high frequency oscillations always are present. After the intersection point, the oscillations will start to increase again with the dynamics remaining similar to that in Fig. 7(d), meaning that it does not leave the charge flipping regime but goes back up into it. For a large enough perturbation $\left(\sum\left|p_{n}\right|^{2} / f \gtrsim 0.8\right.$ for the $\bar{N}=0.16$ vortex) the (approximately) periodic charge flipping dynamics is destroyed.

By changing the coefficients $C_{1}$ and $C_{2}$ in the linear combination of the eigenmode [Eq. (11)], it is possible to obtain other types of dynamics, e.g., translated versions of the dynamics shown in Fig. 7. For some linear combinations, the dynamics will not even become charge flipping, but instead remain similar to that in Fig. 7(b).

Looking at Fig. 4, we can see that the perturbed vortex actually becomes charge flipping slightly before it enters the region between the SDW and dimerlike solution. One may thus obtain a smaller energy difference to the $\kappa= \pm 1$ constant amplitude vortex (with the same norm as the CFV) than Eq. (15), by applying perturbations to the quasiperiodic CFVs. This is because the quasiperiodic CFVs are not the ground state and internal modes may therefore both increase and decrease the energy, which is in contrast to the Peierls-Nabarro barrier for discrete breathers $[48,49]$. Equation $(15)$ is therefore not a strict barrier and should only be considered as an estimation of the true energy barrier. Note that the solution in Fig. 7(c) is located quite close to the SDW solution in Fig. 4.

\section{LINEAR STABILITY}

Linear stability of quasiperiodic solutions is determined by analyzing the Floquet eigenvalues. Due to numerical accuracy, we have set $|\lambda|<1.001$ as the limit for linear stability, $\lambda$ being the largest Floquet eigenvalue.

In the trimer with $C=-1$, both the quasiperiodic family in Sec. V A and the exactly quasiperiodic CFVs in Sec. V C are almost always linearly stable. There are some weak instabilities of the type discussed at the end of Sec. II A, where the Floquet eigenvalues very briefly go out a short distance from the unit circle. The unstable regions found exist in parameter regions (when following a family with fixed norm) of the size $\Delta \bar{H} \lesssim 10^{-5}$, and with eigenvalues $\lambda \lesssim 1.05$.

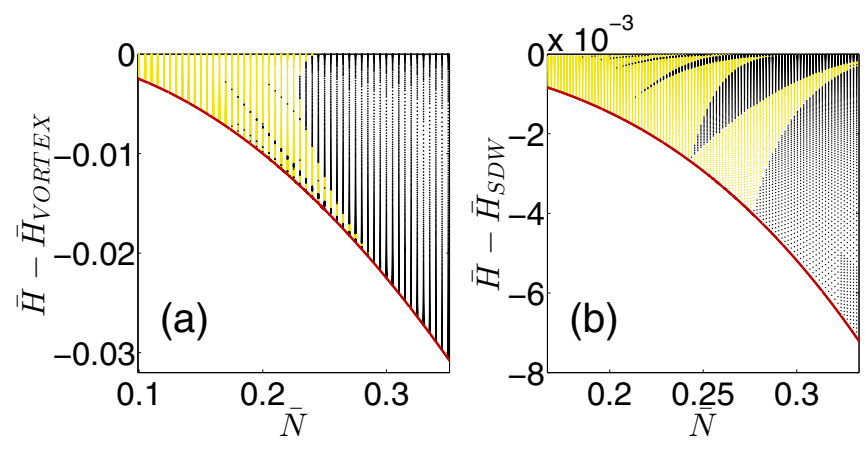

FIG. 8. (Color online) (a) Linear stability of the quasiperiodic solutions of Sec. V A as a function of norm per site and Hamiltonian per site relative to the Hamiltonian per site of the $\kappa= \pm 1$ constant amplitude vortex. (b) Linear stability of the quasiperiodic CFVs of Secs. V B and V C as a function of norm per site and Hamiltonian per site relative to the Hamiltonian per site of the SDW. Both are for the hexamer lattice with $C=1$. Yellow (gray) points indicate linear stability, and black points linear instability. The red (dark gray) curves, which mark the lower boundaries, represent in (a) the SDW solution, and in (b) the dimerlike solution.

Adding a perturbation to these unstable solutions will thus not lead to any drastic changes in the dynamics.

The hexamer on the other hand shows clear instabilities, which thus are of the type that break the antisymmetry. In Fig. 8 we display, in a norm-Hamiltonian per site diagram, which quasiperiodic hexamer solutions that are stable and unstable: Fig. 8(a) is for the quasiperiodic family of Sec. V A, with the Hamiltonian per site being relative to that of the $\kappa= \pm 1$ constant amplitude vortex, while Fig. 8(b) is for the CFV of Sec. V C, with the Hamiltonian per site being relative to that of the SDW solution.

Note that the unstable region for the CFVs that starts on the Dimerlike solution at $\bar{N} \approx 0.27$ is related to the instability of this solution (see Fig. 3). The instabilities in the hexamer, for both quasiperiodic families, are typically of the oscillatory type, where a quadruple of eigenvalues are located in the complex plane off the unit circle, and not along the real axis.

Figure 9 shows a particularly interesting example of the unstable dynamics, where the $\mathrm{CFV}$ at $\bar{H}-\bar{H}_{\text {SDW }}=$ $-1.9 \times 10^{-3}$ and $\bar{N}=0.25$ has been perturbed with a linear combination consisting of the two unstable eigenmodes, the perturbation having the norm $\sum p_{n}^{2}=10^{-6}$. The dynamics on the three additional sites is similar to the ones shown in the plot, i.e., $\psi_{n}(t)$ is similar to $\psi_{n+3}(t)$, but with the amplitude fluctuations in antiphase with each other. There is thus an exchange of norm between the two sublattices, consisting of sites 1 to 3 and sites 4 to 6 , respectively. The instability "kicks in" in bursts of amplitude fluctuations, where the periodic charge flipping is destroyed [see in Fig. 9(c)], but returns after this to the regular behavior with periodic charge flipping. A similar behavior, with periodically reoccurring bursts of amplitude fluctuations, can also be seen for the unstable quasiperiodic solutions of Sec. V A for certain parameters. The dynamics does however not behave like this for all the parameter values for which the solutions are unstable. For other parameters, the unstable dynamics will be dominated by large amplitude oscillations at all times after the instability 

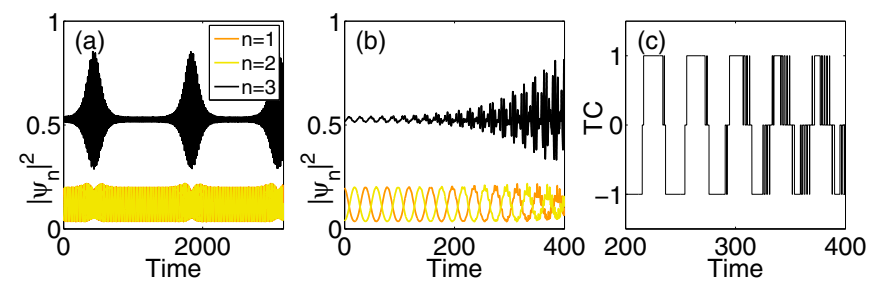

FIG. 9. (Color online) Dynamics of a linearly unstable hexamer CFV with $\bar{H}-\bar{H}_{\text {SDW }}=-1.9 \times 10^{-3}$ and $\bar{N}=0.25$, perturbed with its unstable eigenmodes. Note that only three sites are plotted since the behavior is similar for $\left|\psi_{n}(t)\right|$ and $\left|\psi_{n+3}(t)\right|$, but with the amplitude fluctuations in antiphase. Panels (a) and (b) show $\left|\psi_{n}(t)\right|^{2}$ over two different time scales and panel (c) shows the TC as the instability kicks in. The instability results in bursts of large amplitude fluctuations, where the periodic charge flipping gets destroyed, but it returns to a regular dynamics, with periodic charge flipping, in between the bursts.

has set in. This type of behavior has been studied for the SDW solution in the DNLS trimer (with $C>0$ ) in Ref. [41], where the occurrence of the periodic bursts was shown to be related to the existence of KAM tori which restrict the dynamics to a subpart of phase space.

Note also that since the unstable regimes occur in stripes (Fig. 8), the CFVs that are obtained by perturbing constant amplitude vortices may alternate between being stable and unstable as the magnitude of the perturbation is increased (the $4 l$-site CFVs in Ref. [34] show a similar behavior). Let us finally emphasize that for the values of norm for which the constant amplitude vortex is stable, the overwhelming regime of the hexamer CFVs is stable, and that the CFVs are stable for almost its entire region of existence for the trimer.

\section{CONCLUSIONS}

We have demonstrated numerically that periodically charge flipping vortices exist in trimer and hexamer DNLS models. Below a certain norm, these exist as exact two-frequency quasiperiodic solutions in continuous families, which can be followed between two stationary solutions: the SDW solution and a certain dimerlike solution. These CFVs will, in contrast to the ones in $4 l$-site lattices [34], have periodically oscillating amplitudes. The CFVs that lie close to the SDW can be obtained by perturbing this stationary solution with unstable eigenmodes, resulting essentially in a hole (two holes for the hexamer) that is oscillating between two sites of the lattice, while the CFVs that are close to the dimerlike solution can be obtained by perturbing this stationary solution with stable eigenmodes. Above the threshold norm, the CFV families will bifurcate with other non-charge-flipping, nonstationary solutions.
We have also examined how the CFVs are related to the constant amplitude vortices. As has already been demonstrated in Ref. [39], CFVs can be obtained by applying quite large perturbations, consisting of stable linear eigenmodes, to a $\kappa= \pm 1$ constant amplitude vortex. This is unlike for the $4 l$-site lattices where CFVs occur for infinitesimal perturbations [34]. This implies that the TC for a $\kappa= \pm 1$ constant amplitude vortex should be more stable in the trimer and hexamer, compared to in $4 l$-site lattices. We further explored these CFVs and demonstrated that they can be viewed as perturbed exactly quasiperiodic $\mathrm{CFVs}$, the perturbation resulting in high frequency oscillations in the amplitude, and the behavior of these CFVs can be understood on the basis of the results for the exactly quasiperiodic CFVs.

Since the exactly quasiperiodic CFVs bifurcate with two stationary solutions, it is straightforward to determine the minimum energy difference between a $\kappa= \pm 1$ constant amplitude vortex and an exactly quasiperiodic CFV with the same norm. This may be interpreted as a charge flipping barrier, analogous to the Peierls-Nabarro barrier for translational motion. This is however not a strict barrier, since the appliance of a perturbation, resulting in $\mathrm{CFVs}$ such as the ones mentioned above and studied in Ref. [39], will increase the energy of the CFVs and thereby reduce the energy difference to the $\kappa= \pm 1$ constant amplitude vortex.

For the trimer, the quasiperiodic CFVs are almost always stable, with a few small regions found where weak instabilities occur. For the hexamer, the quasiperiodic CFVs are stable over large parameter regions, but there are also regions where the CFVs experience stronger, oscillatory instabilities. For some parameters, the unstable dynamics will have an interesting behavior where there are periodic "bursts" of large amplitude fluctuations, during which the periodic charge flipping is destroyed, but where it afterwards returns to a regular, periodic charge flipping dynamics.

Since the CFVs exist in continuous families which are stable over large portions of parameter space, and they also can be obtained from quite simple initial conditions (at least in an approximate form), we are convinced that they are realizable in experiments with photonic lattices, multicore fibers, or BECs in optical lattices. We believe that the technique presented recently in Ref. [27] to generate small photonic lattices, and which was used to experimentally study CFVs in four-site lattices, presents an especially promising candidate.

\section{ACKNOWLEDGMENTS}

We acknowledge partial financial support from the Swedish Research Council. Contributions from Nicklas Lagerquist in the initial stages of the work are gratefully acknowledged.
[1] J. F. Nye and M. V. Berry, Proc. R. Soc. London A 336, 165 (1974).

[2] M. M. Salomaa and G. E. Volovik, Rev. Mod. Phys. 59, 533 (1988).
[3] G. Blatter, M. V. Feigel'man, V. B. Geshkanbein, A. I. Larkin, and V. M. Vinokur, Rev. Mod. Phys. 66, 1125 (1994).

[4] J. E. Williams and M. J. Holland, Nature (London) 401, 568 (1999). 
[5] M. R. Matthews, B. P. Anderson, P. C. Haljan, D. S. Hall, C. E. Wieman, and E. A. Cornell, Phys. Rev. Lett. 83, 2498 (1999).

[6] J. R. Anglin and W. Ketterle, Nature (London) 416, 211 (2002).

[7] A. S. Desyatnikov, Y. S. Kivshar, and L. Torner, Prog. Opt. 47, 291 (2005).

[8] M. R. Dennis, K. O’Holleran, and M. J. Padgett, Prog. Opt. 53, 293 (2009).

[9] Z. Dutton and J. Ruostekoski, Phys. Rev. Lett. 93, 193602 (2004).

[10] G. Gibson, J. Courtial, M. J. Padgett, M. Vasnetsov, V. Pas'ko, S. Barnett, and S. Franke-Arnold, Opt. Express 12, 5448 (2004).

[11] J. W. Fleischer, M. Segev, N. K. Efremidis, and D. N. Christodoulides, Nature (London) 422, 147 (2003).

[12] O. Morsch and M. Oberthaler, Rev. Mod. Phys. 78, 179 (2006).

[13] T. Cretegny and S. Aubry, Phys. Rev. B 55, R11929 (1997).

[14] M. Johansson, S. Aubry, Y. B. Gaididei, P. L. Christiansen, and K. Ø. Rasmussen, Physica D 119, 115 (1998).

[15] B. A. Malomed and P. G. Kevrekidis, Phys. Rev. E 64, 026601 (2001).

[16] P. G. Kevrekidis, B. A. Malomed, and Yu. B. Gaididei, Phys. Rev. E 66, 016609 (2002).

[17] J. Yang and Z. H. Musslimani, Opt. Lett. 28, 2094 (2003).

[18] T. J. Alexander, A. A. Sukhorukov, and Y. S. Kivshar, Phys. Rev. Lett. 93, 063901 (2004).

[19] J. Yang, New J. Phys. 6, 47 (2004).

[20] D. E. Pelinovsky, P. G. Kevrekidis, and D. J. Frantzeskakis, Physica D 212, 20 (2005).

[21] M. Öster and M. Johansson, Phys. Rev. E 73, 066608 (2006).

[22] D. N. Neshev, T. J. Alexander, E. A. Ostrovskaya, Y. S. Kivshar, H. Martin, I. Makasyuk, and Z. Chen, Phys. Rev. Lett. 92, 123903 (2004).

[23] J. W. Fleischer, G. Bartal, O. Cohen, O. Manela, M. Segev, J. Hudock, and D. N. Christodoulides, Phys. Rev. Lett. 92, 123904 (2004).

[24] A. Bezryadina, E. Eugenieva, and Z. Chen, Opt. Lett. 31, 2456 (2006).

[25] B. Terhalle, T. Richter, A. S. Desyatnikov, D. N. Neshev, W. Krolikowski, F. Kaiser, C. Denz, and Y. S. Kivshar, Phys. Rev. Lett. 101, 013903 (2008).

[26] B. Terhalle, D. Göries, T. Richter, P. Rose, A. S. Desyatnikov, F. Kaiser, and C. Denz, Opt. Lett. 35, 604 (2010).

[27] F. Diebel, D. Leykam, M. Boguslawski, P. Rose, C. Denz, and A. S. Desyatnikov, Appl. Phys. Lett. 104, 26111 (2014).
[28] J. C. Eilbeck and M. Johansson, in Localization and Energy Transfer in Nonlinear Systems, edited by L. Vásquez, R. S. MacKay, and M. P. Zorzano (World Scientific, New Jersey, 2003), pp. 44-67.

[29] W. Królikowski, U. Trutschel, M. Cronin-Golomb, and C. Schmidt-Hattenberger, Opt. Lett. 19, 320 (1994).

[30] B. Zhu, T. F. Taunay, M. F. Yan, J. M. Fini, M. Fishteyn, E. M. Monberg, and F. V. Dimarcello, Opt. Express 18, 11117 (2010).

[31] F. Y. M. Chan, A. P. T. Lau, and H.-Y. Tam, Opt. Express 20, 4548 (2012)

[32] L. Amico, A. Osterloh, and F. Cataliotti, Phys. Rev. Lett. 95, 063201 (2005).

[33] M. I. Rodas-Verde, H. Michinel, and Y. S. Kivshar, Opt. Lett. 31, 607 (2006).

[34] A. S. Desyatnikov, M. R. Dennis, and A. Ferrando, Phys. Rev. A 83, 063822 (2011).

[35] D. Leykam and A. S. Desyatnikov, Phys. Rev. A 86, 043812 (2012).

[36] L. Casetti and V. Penna, J. Low Temp. Phys. 126, 455 (2002).

[37] D. Leykam and A. S. Desyatnikov, Opt. Lett. 36, 4806 (2011).

[38] M. Johansson and S. Aubry, Nonlinearity 10, 1151 (1997).

[39] N. Lagerquist, bachelor's thesis, Linköping University, 2013; available at http://urn.kb.se/resolve?urn=urn:nbn:se:liu:diva111092.

[40] C. Robinson, Dynamical Systems, 2nd ed. (CRC Press, Boca Raton, 1999).

[41] M. Johansson, J. Phys. A: Math. Gen. 37, 2201 (2004).

[42] J. Carr and J. C. Eilbeck, Phys. Lett. A 109, 201 (1985).

[43] J. C. Eilbeck, P. S. Lomdahl, and A. C. Scott, Physica D 16, 318 (1985).

[44] R. Franzosi and V. Penna, Phys. Rev. E 67, 046227 (2003).

[45] J. C. Eilbeck, in Physics of Many-Particle Systems, edited by A. Davydov (Naukova Dumka, Kiev, 1987), Vol. 12, p. 41.

[46] C. García-Azpeitia and J. Ize, Discrete and Continuous Dynamical Systems, Series S 6, 975 (2013).

[47] T. J. Alexander, D. Yan, and P. G. Kevrekidis, Phys. Rev. E 88, 062908 (2013).

[48] J. C. Eilbeck, in Computer Analysis for Life Science: Progress and Challenges in Biological and Synthetic Polymer Research, edited by C. Kawabata and A. R. Bishop (Ohmsha, Tokyo, 1986), pp. 12-21.

[49] M. Johansson and P. Jason, arXiv:1412.0994 [in Quodons in Mica: Nonlinear Localized Traveling Excitations in Crystals, edited by J. F. R. Archilla et al. (Springer, to be published 2015)]. 